\title{
LARGE-TIME BEHAVIOR OF SOLUTIONS TO A SCALAR CONSERVATION LAW IN SEVERAL SPACE DIMENSIONS
}

\author{
PATRICIA BAUMAN ${ }^{1}$ AND DANIEL PHILLIPS ${ }^{2}$
}

\begin{abstract}
We consider solutions of the Cauchy problem in $\mathbf{R}_{+}^{n+1}$ for the equation $u_{t}+\operatorname{div}_{x} f(u)=0$. The initial data is assumed to be a compact perturbation of a function of the form, $\varphi(x)=a$ for $\langle x, \mu\rangle>0, \varphi(x)=b$ for $\langle x, \mu\rangle\langle 0$, where $a$ and $b$ are constants and $\mu$ is a given unit vector. The Cauchy problem together with an entropy condition on $u$ is known to be well posed. The solution with unperturbed initial data, $\varphi(x)$, is a traveling shock, $\varphi(x-\vec{k} t)$, provided that $\varphi(x-\vec{k} t)$ satisfies the entropy condition (an inequality on $a, b, \mu$, and $f$ ). Assuming this type of condition on $\varphi$, we study the largetime behavior of $u$. In particular, we show that $u$ converges to a traveling shock whose profile agrees with $\langle x, \mu\rangle=0$ outside of a compact set.
\end{abstract}

Introduction. In $1958 \mathrm{~A}$. M. Ilin and O. A. Oleinik [4] studied the large-time stability of a shock solution to a single conservation law. They considered the Cauchy problem

$$
\begin{gathered}
\frac{\partial u}{\partial t}+\frac{\partial}{\partial x} f(u)=0 \text { for } t>0, x \in \mathbf{R} \\
u(0, x)=u_{0}(x) \text { for } x \in \mathbf{R}
\end{gathered}
$$

where $u_{0}$ is bounded,

$$
u_{0}(x)=a \cdot \chi_{\mathbf{R}^{+}}(x)+b \cdot \chi_{\mathbf{R}^{-}}(x) \equiv \psi(x)
$$

for $|x|$ sufficiently large,

$$
a<b \text { and } f^{\prime \prime}>0 .
$$

Solutions of the Cauchy problem are unique among those which satisfy an entropy condition (see $(\mathrm{E})$ in $\S 1$ ). The structure assumptions, $(0.3)$, ensure that $\psi(x-k t)$ is a solution of $(0.1)$ and satisfies the entropy condition when $k=(f(b)-f(a)) /(b-a)$. Ilin and Oleinik used the viscosity method to show that for all $t$ sufficiently large, $u(t, x)=\psi\left(x_{0}+x-k t\right)$ for some $x_{0} \in \mathbf{R}$. Thus if the initial data is a compact perturbation of $\psi$, the solution evolves after finite time into the traveling shock solution, $\psi\left(x_{0}+x-k t\right)$. We remark that the same result was proved by C. Dafermos [2] and T. P. Liu [7] using the notion of generalized characteristic curves.

In this paper we investigate the $n$-dimensional analog of this problem:

$$
\begin{gathered}
\partial u / \partial t+\operatorname{div} f(u)=0 \text { for } t>0, x \in \mathbf{R}^{n}, \\
u(0, x)=u_{0}(x) \text { for } x \in \mathbf{R}^{n}
\end{gathered}
$$

Received by the editors December 13, 1985.

1980 Mathematics Subject Classification. Primary 35L65, 35B40.

${ }^{1}$ Research supported in part by a Purdue Research Foundation XL Grant.

${ }^{2}$ Research supported in part by N.S.F. Grant No. DMS-8201036. 
where $f \in C^{1}\left(\mathbf{R} ; \mathbf{R}^{n}\right)$ and $n \geq 1$. We assume that $u_{0} \in L^{\infty}\left(\mathbf{R}^{n}\right)$ and $u_{0}=\varphi$ for $|x| \geq R_{1}>0$, where

$$
\varphi(x)= \begin{cases}a & \text { for }\langle x, \mu\rangle>0 \\ b & \text { for }\langle x, \mu\rangle<0\end{cases}
$$

for some unit vector $\mu \in \mathbf{R}^{n}$ and $a<b$. We define a solution of (0.4) to be a distribution solution in $C\left([0, \infty) ; L_{\text {loc }}^{1}\left(\mathbf{R}^{n}\right)\right)$ which satisfies Vol'pert's entropy condition, (E). (See §1.)

If $k=[f(b)-f(a)] /(b-a)$, it follows from $(E)$ that $\varphi(x-k t)$ is a solution of (0.4) if and only if $\langle\tilde{f}(c), \mu\rangle \leq 0$ for $a \leq c \leq b$ where $\tilde{f}(c) \equiv f(c)-f(a)-(c-a) k$. We assume throughout the paper that $\varphi$ is nondegenerate, i.e., the above entropy condition holds in the following strict sense: there exists a constant $\theta>0$ such that

$$
\langle\mu, \tilde{f}(c)\rangle \leq-\theta(b-c)(c-a) \text { for } a \leq c \leq b .
$$

We summarize our results as follows. In $\S \S 1$ and 2 we show that there are bounds $m_{1}<a$ and $m_{2}>b$ (determined explicitly by $\tilde{f}$ ) so that if

$$
m_{1}<\operatorname{ess} \inf u_{0} \text { and } m_{2}>\text { ess } \sup u_{0},
$$

then after a finite time, $a \leq u(t, x) \leq b$. Moreover, there is a set $M \subset \mathbf{R}^{n}$ and a constant $R_{2}>0$ such that if

$$
v(x)=b \cdot \chi_{M}(X)+a \cdot \chi_{\mathbf{R}^{n}-M}(x),
$$

then

$$
u(t, x)-v(x-k t) \rightarrow 0 \text { in } L^{1}\left(\mathbf{R}^{n}\right) \text { as } t \rightarrow \infty
$$

and

$$
M \cap\left\{x:|x|>R_{2}\right\}=\left\{x:\langle x, \mu\rangle<0,|x|>R_{2}\right\} .
$$

Our investigation shows that the large-time behavior of $u$ can be described more definitely and that it depends on $d \equiv \operatorname{dimension}(\operatorname{span}\{\tilde{f}(c): a \leq c \leq b\})$. In $\S 3$ we study the case $d=n$ and prove the following results. Here the initial data is called admissible if it satisfies (0.7) and $u_{0}=\varphi$ for $|x| \geq R_{1}>0$.

THEOREM 3.2. If $u_{0}$ is admissible and $d=n$, then $M$ is a Lipschitz domain. More precisely, there is a set of coordinates $\left(y_{1}, \ldots, y_{n}\right)=\left(\hat{y}, y_{n}\right)$ obtained by a rotation and a constant, $C$, (both depending only on $\tilde{f})$ so that $M=\left\{\left(\hat{y}, y_{n}\right): y_{n}<\right.$ $g(\hat{y})\}$ where $g$ is Lipschitz continuous and $\|\nabla g\|_{\infty} \leq C .{ }^{3}$

We prove the following stability estimate for the shock front, $g$.

THEOREM 3.3. Suppose $u_{1}$ and $u_{2}$ are admissible initial data and $d=n$. Let $g_{1}$ and $g_{2}$ be the end state shock fronts. Then

$$
\sup _{\hat{y} \in \mathbf{R}^{n-1}}\left|g_{1}(\hat{y})-g_{2}(\hat{y})\right| \leq C(\tilde{f}) \cdot\left(\int_{\mathbf{R}^{n}}\left|u_{1}-u_{2}\right| d x\right)^{1 / n} .
$$

Using the above estimate, we show that in finite time, $u$ is equal to the traveling shock, $v(x-k t)$, outside an arbitrarily small neighborhood of the shock front.

\footnotetext{
${ }^{3}$ For $n=1, g$ should be replaced by a constant, $x_{0}$, and $M=\left\{x: x<x_{0}\right\}$.
} 
THEOREM 3.12. Suppose $d=n$ and $u_{0}$ is admissible and piecewise continuous. (See 3.5.) If $\varepsilon>0$ there exists $T_{\varepsilon}>0$ such that $u(t, x)=v(x-k t)$ for $t \geq T_{\varepsilon}$ and $\operatorname{dist}(x-k t, \partial M)>\varepsilon$.

In $\S 4$ we study the case $d<n$ and show that it reduces to the case described above. Without loss of generality we assume that $V \equiv \operatorname{span}\{\tilde{f}(c): a \leq c \leq b\}=$ $\mathbf{R}^{d} \times\{0\}$; the projection of $\mu$ on $V$ is nontrivial; and its unit-length normalization, $\left(\mu^{\prime}, 0\right)$, satisfies

$$
\left\langle\left(\mu^{\prime}, 0\right), \tilde{f}(c)\right\rangle \leq-\theta(b-c)(c-a) \text { for } a \leq c \leq b .
$$

(This holds after an appropriate change of coordinates.)

The problem, (0.4) and (0.5), can then be interpreted (after a finite time) as a parametrized set of problems in $d$ space dimensions. To describe this in more detail, let us denote $\left(x_{1}, \ldots, x_{n}\right)$ by $\left(x^{\prime}, x^{\prime \prime}\right)$ with $x^{\prime} \in \mathbf{R}^{d}$ and $x^{\prime \prime} \in \mathbf{R}^{n-d}$. We prove

LEMMA 4.1. Suppose $u_{0}$ is admissible and $d<n$. Under the above hypotheses on $\tilde{f}$, there exists $T>0$ (explicitly computable from $\tilde{f}$ ) so that if $t \geq T$,

$$
u(t, x)=\tilde{u}\left(t, x^{\prime}-k^{\prime} t ; x^{\prime \prime}-k^{\prime \prime} t\right) .
$$

Here $\tilde{u}\left(t, x^{\prime} ; x^{\prime \prime}\right)$ is defined for a.e. $x^{\prime \prime} \in \mathbf{R}^{n-d}$ as the solution of the problem

$$
\begin{gathered}
\frac{\partial \tilde{u}}{\partial t}+\sum_{i=1}^{d} \frac{\partial}{\partial x_{i}}\left(\tilde{f}_{i}(\tilde{u})\right)=0 \quad \text { for } t \geq T ; x^{\prime} \in \mathbf{R}^{d}, \\
\tilde{u}\left(T, x^{\prime} ; x^{\prime \prime}\right)=u\left(T, x^{\prime}+k^{\prime} T, x^{\prime \prime}+k^{\prime \prime} T\right) .
\end{gathered}
$$

The function $u\left(T, x^{\prime}+k^{\prime} T, x^{\prime \prime}+k^{\prime \prime} T\right)$ for fixed $x^{\prime \prime} \in \mathbf{R}^{n-d}$ is admissible relative to a nondegenerate shock defined through $\mu^{\prime}$.

From our results for the case $d=n$, we have

THEOREM 4.6. Suppose $u_{0}$ is admissible, piecewise continuous, and $d<n$. For all $\varepsilon>0$ and a.e. $z^{\prime \prime} \in \mathbf{R}^{n-d}$ there exists $T=T\left(\varepsilon, z^{\prime \prime}\right)>0$ and a Lipschitz domain, $M\left(z^{\prime \prime}\right)$, in $\mathbf{R}^{d}$ such that $\chi_{M}\left(z^{\prime}, z^{\prime \prime}\right)=\chi_{M\left(z^{\prime \prime}\right)}\left(z^{\prime}\right)$ and if $x^{\prime \prime}-k^{\prime \prime} t=z^{\prime \prime}$,

$$
\begin{aligned}
u\left(t, x^{\prime}, x^{\prime \prime}\right) & =v\left(t, x^{\prime}-k^{\prime} t, x^{\prime \prime}-k^{\prime \prime} t\right) \\
& =b \cdot \chi_{M\left(z^{\prime \prime}\right)}\left(x^{\prime}-k^{\prime} t\right)+a \cdot \chi_{\mathbf{R}^{d}-M\left(z^{\prime \prime}\right)}\left(x^{\prime}-k^{\prime} t\right)
\end{aligned}
$$

for $t \geq T$ and $\operatorname{dist}\left(x^{\prime}-k^{\prime} t, \partial M\left(z^{\prime \prime}\right)\right) \geq \varepsilon$.

Solutions of (0.4) and (0.5) when $n=2$ and $u_{0}$ is constant in each of the four quadrants were studied by D. Wagner in [9]. He assumed that $f=\left(f_{1}, f_{2}\right)$ with $f_{1}^{\prime \prime}, f_{2}^{\prime \prime}>0$ and $f_{1}$ sufficiently close to $f_{2}$. Under these assumptions he constructed the solution and analyzed its qualitative properties. Further study of this problem was done by W. B. Lindquist [6] assuming that $f_{1}=f_{2}$ and $f_{1}$ has at most two inflection points.

In [1] E. Conway studied the large-time behavior of solutions of (0.4) and (0.5) in $n$ space dimensions assuming that $u_{0}$ has compact support and $f$ is convex in some direction. He proved decay estimates on the $L^{\infty}$-norm of such solutions.

As far as we know, all previous studies of the large-time behavior of $u$ assumed some type of convexity condition on $f$. Our primary assumption is (0.6). In particular, our results are new even for the case $n=1$. 
1. Super and subentropy solutions. In this section we define a notion of super and subentropy solutions of a conservation law. A technique used throughout the paper is the explicit construction of such functions to obtain estimates on $u(t, x)$ for large time.

First, let us recall some results proved by Vol'pert in [8]. Suppose $u \in B V\left(\mathbf{R}_{+}^{n+1}\right)$ $\cap L^{\infty}\left(\mathbf{R}_{+}^{n+1}\right)$. Then Vol'pert showed that with the exception of a set of $H^{n}$-measure zero every $(t, x)$ in $\mathbf{R}^{n+1}$ is a regular point of $u$. This means that there is a unit vector, $\nu$, in $\mathbf{R}^{n+1}$ and numbers $l_{\nu} u(t, x)$ and $l_{-\nu} u(t, x)$ such that for each $\varepsilon>0$

$$
\lim _{r \rightarrow 0} \frac{\mid\left\{(\tau, y) \in B_{r}(t, x):\left|u(\tau, y)-l_{ \pm \nu} u(t, x)\right|>\varepsilon \text { and }\langle(\tau-t, y-x), \pm \nu\rangle \geq 0\right\} \mid}{\left|B_{r}(t, x)\right|}=0
$$

(Here $|E|$ denotes the Lebesgue measure of a set, $E$.) We denote the set of regular points where $l_{\nu} u(t, x) \neq l_{-\nu} u(t, x)$ as the set of approximate jump points, $\Gamma(u)$. We remark that up to a multiple of $\pm 1, \nu$ is unique for $(t, x) \in \Gamma(u)$ and is called a normal to $\Gamma(u)$ at $(t, x)$. We adopt the convention that $u^{+}=l_{\nu} u(t, x), u^{-}=$ $l_{-\nu} u(t, x)$, and $\nu$ is chosen so that $u_{+} \leq u_{-}$. If $g(t)$ is a continuously differentiable function, Vol'pert proved that $g(u(t, x)) \in B V\left(\mathbf{R}_{+}^{n+1}\right)$ and $\nabla g(u)=\tilde{g}(u) \cdot \nabla u$ as a Borel measure, where for each regular point, $(t, x)$,

$$
\tilde{g}(u)= \begin{cases}\frac{g\left(u^{+}\right)-g\left(u^{-}\right)}{u^{+}-u^{-}} & \text {if }(t, x) \in \Gamma(u), \\ g^{\prime}(\bar{u}(t, x)) & \text { if }(t, x) \notin \Gamma(u)\end{cases}
$$

and $\bar{u}(t, x)=\lim _{r \rightarrow 0} f_{B_{r}(t, x)} u(\tau, y) d \tau d y$, the Lebesgue limit of $u$ at $(t, x)$. (This is well defined $H^{n}$-almost everywhere.)

Vol'pert proved that solutions of $(0.4)$ and (0.5) are unique among those which satisfy an entropy condition. More precisely, suppose $u_{0} \in B V\left(\mathbf{R}^{n}\right) \cap L^{\infty}\left(\mathbf{R}^{n}\right)$. Let $A(u)=(u, f(u))$ and define $S: \mathbf{R}^{2} \rightarrow \mathbf{R}^{n+1}$ by

$$
S(u, v)=(A(u)-A(v)) \cdot \operatorname{sign}(u-v) .
$$

Then there is a unique function $u \in B V\left(\mathbf{R}_{+}^{n+1}\right)$ satisfying

$$
\begin{aligned}
& \partial u / \partial t+\operatorname{div} f(u)=\operatorname{div} A(u)=0, \\
& \lim _{t \rightarrow 0+} \bar{u}(t, x)=u_{0}(x) \text { for } L^{n} \text {-a.e. } x
\end{aligned}
$$

and the entropy condition:

$$
\operatorname{div} S(u, c) \leq 0 \text { for all } c \in \mathbf{R} \text { as a distribution on } \mathbf{R}_{+}^{n+1} .
$$

When $u \in B V\left(\mathbf{R}_{+}^{n+1}\right) \cap L^{\infty}\left(\mathbf{R}_{+}^{n+1}\right)$ and $u$ satisfies (1.1), the entropy condition, $(E)$, is equivalent to

$\left(\mathrm{E}^{\prime}\right) \quad\left\langle S\left(u^{+}, c\right), \nu\right\rangle \leq\left\langle S\left(u^{-}, c\right), \nu\right\rangle$ for all $c \in \mathbf{R}$ and $H^{n}$-almost every point in the jump set, $\Gamma(u)$.

In addition, Vol'pert proved that if $u_{0} \in B V\left(\mathbf{R}^{n}\right) \cap L^{\infty}\left(\mathbf{R}^{n}\right)$, then

(i) $\bar{u}(t, x) \in C\left([0, \infty) ; L_{\text {loc }}^{1}\left(\mathbf{R}^{n}\right)\right)$,

(ii) $|\bar{u}| \leq\left\|u_{0}\right\|_{\infty}$,

(iii) For any two solutions, $u$ and $w$, of $(1.1)$ which satisfy $(E)$ and such that $u, w \in L^{\infty}\left(\mathbf{R}_{+}^{n+1}\right) \cap C\left([0, \infty) ; L_{\text {loc }}^{1}\left(\mathbf{R}^{n}\right)\right)$ and $|u|,|w| \leq m<\infty$, we have

$$
\int_{B_{r}}|\bar{u}(t, x)-\bar{w}(t, x)| d x \leq \int_{B_{r+l t}}|u(x, 0)-w(x, 0)| d x
$$

where $l=\left(\sum_{i=1}^{n}\left(\max _{|u| \leq m}\left|f_{i}^{\prime}(u)\right|\right)^{2}\right)^{1 / 2}$. 
If $u_{0} \in L^{\infty}\left(\mathbf{R}^{n}\right)$, Vol'pert showed that there is a distribution solution of (1.1) and (1.2) in the class $C\left([0, \infty) ; L_{\text {loc }}^{1}\left(\mathbf{R}^{n}\right)\right)$ which satisfies (E) and also satisfies (ii) and (iii). Kruzkov proved that it is unique in a broader class [5].

As stated in the introduction, we define a solution of (1.1) to be a function, $u$, in $C\left([0, \infty) ; L_{\text {loc }}^{1}\left(\mathbf{R}^{n}\right)\right)$ which satisfies $(1.1)$ in the sense of distributions and also satisfies the entropy condition (E).

We define super and subentropy solutions as follows:

DEFINITION 1.4. Suppose

$$
u(t, x) \in B V\left(\mathbf{R}_{+}^{n+1}\right) \cap L^{\infty}\left(\mathbf{R}_{+}^{n+1}\right) \cap C\left([0, \infty) ; L_{\text {loc }}^{1}\left(\mathbf{R}^{n}\right)\right) .
$$

Then $u$ is a super $(s u b)$ solution of (1.1) if

$$
\begin{aligned}
\text { (1) } \operatorname{div} A(u) & \left.\equiv\left\langle\left[A\left(u^{+}\right)-A\left(u^{-}\right)\right], \nu\right\rangle d \nvdash^{n}\right|_{\Gamma(u)}+\left.\left(u_{t}+\sum_{i=1}^{n} f_{i}^{\prime}(u) \cdot u_{x_{i}}\right)\right|_{\Gamma(u)^{c}} \\
& \geq 0(\leq 0) .
\end{aligned}
$$

(2) For $\not^{n}$-a.e. $(t, x) \in \Gamma(u)$,

$$
\begin{aligned}
& \left\langle A\left(u^{+}\right), \nu\right\rangle \geq(A(c), \nu) \quad \text { for } u^{+} \leq c \leq u^{-} \\
& \left.\quad \text { (sub: }\left\langle A\left(u^{-}\right), \nu\right\rangle \geq\langle A(c), \nu\rangle \text { for } u^{+} \leq c \leq u^{-}\right) .
\end{aligned}
$$

The inequalities in condition (2) are splitting of the entropy condition into two inequalities.

The following lemma will be used to prove a maximum principle for super and subsolutions. We use the notation $\sigma^{+}(z)=\chi_{\mathbf{R}^{+}}(z), \sigma^{-}(z)=-\chi_{\mathbf{R}^{-}}(z)$, and $S^{ \pm}(u, v)=[A(u)-A(v)] \cdot \sigma^{ \pm}(u-v)$.

LEMMA 1.5. Suppose $u \in B V\left(\mathbf{R}_{+}^{n+1}\right) \cap L^{\infty}\left(\mathbf{R}_{+}^{n+1}\right) \cap C\left([0, \infty) ; L_{\text {loc }}^{1}\left(\mathbf{R}^{n}\right)\right)$. Then $u$ is a supersolution iff $\operatorname{div} S^{-}(u, c) \leq 0$ for all $c \in \mathbf{R} ; u$ is a subsolution iff $\operatorname{div} S^{+}(u, c) \leq 0$ for all $c \in \mathbf{R}$.

Proof. Let $\Delta S^{-}(u, c)=\left[S^{-}\left(u^{+}, c\right)-S^{-}\left(u^{-}, c\right)\right]$ in $\Gamma(u)$. Then from the calculus of $B V$ functions $[8, \S 15]$ we have

$$
\left.\operatorname{div} S^{-}(u, c)=\left.\overline{\sigma^{-}}(u-c) \cdot \operatorname{div} A(u)\right|_{\Gamma(u)^{c}}+\left\langle\Delta S^{-}(u, c), \nu\right\rangle d\right\}\left.^{n}\right|_{\Gamma(u)} .
$$

Note first that $\left\langle\Delta S^{-}(u, c), \nu\right\rangle \leq 0$ a.e. on $\Gamma(u)$ for $c \in \mathbf{R}$ iff $\langle A(c), \nu\rangle \leq\left\langle A\left(u^{+}\right), \nu\right\rangle$ for $u^{+} \leq c \leq u^{-}$a.e. on $\Gamma(u)$. Second, if we choose $c>\|u\|_{\infty}$, then $\overline{\sigma^{-}}(u-c) \equiv-1$. Thus

$$
\left.\overline{\sigma^{-}}(u-c) \cdot \operatorname{div} A(u)\right|_{\Gamma(u)^{c}} \leq 0 \text { for all } c \in \mathbf{R}
$$

is equivalent to $\operatorname{div} A(u) \geq 0$ in $\Gamma(u)^{c}$. This shows that $\operatorname{div} S^{-}(u, c) \leq 0$ iff $u$ is a supersolution. The result for subsolutions is proved similarly.

THEOREM 1.6. Suppose $u$ is a supersolution and $v$ is a subsolution. Let

$$
m=\sup \left\{\max \{|u(t, x)|,|v(t, x)|\}: x \in \mathbf{R}^{n}, 0 \leq t \leq T\right\}
$$

and

$$
l=\left(\sum_{i=1}^{n}\left(\max _{|u| \leq m}\left|f_{i}^{\prime}(u)\right|\right)^{2}\right)^{1 / 2}
$$


Then $\operatorname{div} S^{-}(u, v)=\operatorname{div} S^{+}(v, u) \leq 0$ and for $0 \leq t \leq T<\infty$,

$$
\int_{B_{r}} \max \{0, v-u\}(t, x) d x \leq \int_{B_{r+l t}} \max \{0, v-u\}(0, x) d x .
$$

Proof. We have from $[8, \S 15]$ that

$$
\operatorname{div} S^{-}(u, v)=\left.\overline{\sigma^{-}}(u-v) \cdot \operatorname{div}[A(u)-A(v)]\right|_{\Gamma(u-v)^{c}}+\left.\left\langle\Delta S^{-}(u, v), \nu\right\rangle d \not^{n}\right|_{\Gamma(u-v)}
$$

where $\Delta S^{-}(u, v)=S^{-}\left(l_{\nu} u, l_{\nu} v\right)-S^{-}\left(l_{-\nu} u, l_{-\nu} v\right)$ and $\nu$ is the normal to $\Gamma(u-v)$. The first term is a nonpositive measure. As for the second term, it follows from Lemma 1.5 that for any $c$

(1) $\left\langle S^{-}\left(l_{\nu} u, c\right), \nu\right\rangle \leq\left\langle S^{-}\left(l_{-\nu} u, c\right), \nu\right\rangle$ and

(2) $\left\langle S^{+}\left(l_{\nu} v, c\right), \nu\right\rangle \leq\left\langle S^{+}\left(l_{-\nu} v, c\right), \nu\right\rangle$,

$\gamma^{n}$-almost everywhere on $\Gamma(u-v)$. Set $c=l_{\nu} v$ in (1) and $c=l_{-\nu} u$ in (2). Since $S^{+}(h, g)=S^{-}(g, h)$, it follows from (1) and (2) that $\Delta S^{-}(u, v) \leq 0$ a.e. on $\Gamma(u-v)$. This proves that $\operatorname{div} S^{-}(u, v)=\operatorname{div} S^{+}(v, u) \leq 0$.

The second assertion follows from Green's theorem on the region, $D=\{(x, \tau):|x|$ $<r+l(t-\tau), 0<\tau<t\}$. We have (from $[8, \S 14]$ )

$$
\begin{aligned}
0 \geq & \int_{D} \operatorname{div} S^{-}(u, v) d \tau d x=\int_{B_{r}} \max \{0, v-u\}(t, x) d x \\
& -\int_{B_{r+l t}} \max \{0, v-u\}(0, x) d x \\
& +\int_{\partial D \cap\{0<\tau<t\}}\left\langle S^{-}(l(u), l(v)), N\right\rangle d \sharp^{n}
\end{aligned}
$$

where $l(u), l(v)$ are the inward traces of $u, v$ and $N$ is the outward-pointing normal on $\partial D$. From the choice of $l$ the third term is nonnegative. This proves the theorem.

REMARK 1.7. The above theorem also holds if $v \notin B V\left(\mathbf{R}_{+}^{n+1}\right)$ but $v \in$ $L^{\infty}\left(\mathbf{R}_{+}^{n+1}\right) \cap C\left([0, \infty) ; L_{\text {loc }}^{1}\left(\mathbf{R}^{n}\right)\right)$ and $v$ is a solution of $(1.1)$. This follows from Vol'pert's construction of $v$ as a limit in $C\left([0, \infty) ; L_{\text {loc }}^{1}\left(\mathbf{R}^{n}\right)\right)$ of a sequence of functions, $\left\{v_{j}(t, x)\right\}$, which are solutions of $(1.1)$ and satisfy the hypotheses of Theorem 1.6 .

2. Preliminary results. In this section we prove preliminary results concerning the large-time behavior of solutions of (1.1) and (1.2). We assume throughout the paper that $f \in C^{1}\left(\mathbf{R} ; \mathbf{R}^{n}\right)$ for $n \geq 1, u(t, x)$ is a solution of $(1.1)$ and $(1.2)$, and $u_{0}$ is a compact perturbation of the Riemann data,

$$
\varphi(x)= \begin{cases}a & \text { for }\langle x, \mu\rangle>0, \\ b & \text { for }\langle x, \mu\rangle<0\end{cases}
$$

where $\mu$ is a unit vector in $\mathbf{R}^{n}$ and $a<b$.

We define

$$
k=[f(b)-f(a)] /(b-a) \quad \text { and } \quad \tilde{f}(c)=f(c)-f(a)-(c-a) k .
$$

Our main assumption (involving $f$ and $\varphi$ ) is that $\varphi$ is nondegenerate; that is, there exists a constant $\theta>0$ such that

$$
\langle\tilde{f}(c), \mu\rangle \leq-\theta(b-c)(c-a) \text { for } a \leq c \leq b .
$$


This is essentially a strict entropy condition on $\varphi(x-k t)$. To check this, we note that $w(t, x) \equiv \varphi(x-k t)$ is in $B V\left(\mathbf{R}_{+}^{n+1}\right) \cap L^{\infty}\left(\mathbf{R}_{+}^{n+1}\right)$ and is a weak solution of (1.1). The entropy condition (E) on $w$ is equivalent to $\left(\mathrm{E}^{\prime}\right)$ which can be stated in the form

$$
\left\langle A(c)-A\left(w^{+}\right), \nu\right\rangle \leq\left\langle A\left(w^{-}\right)-A(c), \nu\right\rangle \text { for } w^{+} \leq c \leq w^{-}
$$

$H^{n}$-a.e. in $\Gamma(w)$. (See $\S 1$.) Since $\nu$ is in the same direction as $(\langle-k, \mu\rangle, \mu)$ and $\tilde{f}(a)=\tilde{f}(b)=0,\left(\mathrm{E}^{\prime \prime}\right)$ reduces to

$$
\langle\tilde{f}(c), \mu\rangle \leq 0 \text { for } a \leq c \leq b .
$$

Thus (2.1) ensures that $\varphi(x-k t)$ is a traveling wave solution of (1.1).

We define

$$
m_{1}=\inf \left\{m: m<a \text { and } \alpha_{1}(m) \equiv \max _{m \leq c \leq a} \frac{\langle\tilde{f}(c), \mu\rangle}{c-a}<\beta_{1}(m) \equiv \min _{m \leq c \leq a} \frac{\langle\tilde{f}(c), \mu\rangle}{c-b}\right\}
$$

and

$$
m_{2}=\sup \left\{m: m>b \text { and } \alpha_{2}(m) \equiv \max _{b \leq c \leq m} \frac{\langle\tilde{f}(c), \mu\rangle}{c-a}<\beta_{2}(m) \equiv \min _{b \leq c \leq m} \frac{\langle\tilde{f}(c), \mu\rangle}{c-b}\right\} .
$$

By (2.1) and the differentiability of $f, m_{1}$ and $m_{2}$ are well defined with $-\infty \leq$ $m_{1}<a$ and $b<m_{2} \leq+\infty$. The initial data, $u_{0}$, is called admissible if

(i) for some $R_{1}>0, u_{0}(x)=\varphi(x)$ for $|x| \geq R_{1}$, and

(ii) $u_{0} \in L^{\infty}\left(\mathbf{R}^{n}\right), m_{1}<$ ess $\inf u_{0}$, and $m_{2}>$ ess sup $u_{0}$.

We first prove that if $u_{0}$ is admissible then for some $t^{*}<\infty$, we have $a \leq$ $u(t, x) \leq b$ for $t \geq t^{*}$. It will be convenient in the proof to assume (without loss of generality) that $f(a)=f(b)=0$ and hence $k=0$. This is possible because if $k \neq 0$ we can make a change of variables,

$$
x_{1}=x-k t, \quad t_{1}=t,
$$

and set $\tilde{u}\left(t_{1}, x_{1}\right)=u(t, x)$. Equations (1.1), (1.2), and the entropy condition (E) become

$$
\partial \tilde{u} / \partial t_{1}+\operatorname{div}_{x_{1}} \tilde{f}(\tilde{u})=0, \quad \tilde{u}(0, x)=u_{0}(x)
$$

and

$$
\operatorname{div}_{\left(t_{1}, x_{1}\right)} \tilde{S}(\tilde{u}, c) \leq 0 \text { for all } c \in \mathbf{R}
$$

where $\tilde{S}(v, w)=\operatorname{sign}(v-w) \cdot[\tilde{A}(v)-\tilde{A}(w)]$ and $\tilde{A}(v)=(v, \tilde{f}(v))$. Thus the effect is to replace $f$ by $\tilde{f}$ which satisfies $\tilde{f}(a)=\tilde{f}(b)=0$.

THEOREM 2.3. If $u_{0}$ is admissible, there exists $t^{*}<\infty$ (defined below) such that $a \leq u(t, x) \leq b$ for $t \geq t^{*}$.

ProOF. Let $b^{\prime}=\operatorname{ess} \sup u_{0}$ and $a^{\prime}=\operatorname{ess}$ inf $u_{0}$. Define

$$
\begin{aligned}
& t_{1}= \begin{cases}0 & \text { if } a^{\prime} \geq a, \\
2 R_{1} /\left[\beta_{1}\left(a^{\prime}\right)-\alpha_{1}\left(a^{\prime}\right)\right] & \text { if } a^{\prime}<a,\end{cases} \\
& t_{2}= \begin{cases}0 & \text { if } b^{\prime} \leq b, \\
2 R_{1} /\left[\beta_{2}\left(b^{\prime}\right)-\alpha_{2}\left(b^{\prime}\right)\right] & \text { if } b^{\prime}>b,\end{cases}
\end{aligned}
$$

and let $t^{*}=\max \left\{t_{1}, t_{2}\right\}$. 
First we show that $u \leq b$ for $t \geq t^{*}$. We assume that $b^{\prime}>b$ because if not, $u \leq b$ for all time by the maximum principle. We also assume (w.l.o.g.) that $f(a)=f(b)=0$ and hence $f=\tilde{f}$.

Let $\alpha=\alpha_{2}\left(b^{\prime}\right), \beta=\beta_{2}\left(b^{\prime}\right)$, and consider the function defined by

$$
w(t, x)= \begin{cases}b & \text { for }\langle x, \mu\rangle<\beta t-R_{1}, \\ b^{\prime} & \text { for } \beta t-R_{1}<\langle x, \mu\rangle<\alpha t+R_{1}, \\ a & \text { for } \alpha t+R_{1}<\langle x, \mu\rangle,\end{cases}
$$

when $0 \leq t \leq 2 R_{1} /(\beta-\alpha)$;

$$
w(t, x)= \begin{cases}b & \text { for }\langle x, \mu\rangle<2 R_{1} \alpha /(\beta-\alpha)+R_{1}, \\ a & \text { for } 2 R_{1} \alpha /(\beta-\alpha)+R_{1}<\langle x, \mu\rangle,\end{cases}
$$

when $t>2 R_{1} /(\beta-\alpha)$.

We claim that $w$ is a supersolution. Clearly $w \in B V\left(\mathbf{R}_{+}^{n+1}\right) \cap L^{\infty}\left(\mathbf{R}_{+}^{n+1}\right) \cap$ $C\left([0, \infty) ; L_{\text {loc }}^{1}\left(\mathbf{R}^{n}\right)\right)$ and since $w$ is piecewise constant we need only verify (1.4.2) on $\Gamma(w)$.

On the jump from $w=b$ to $w=b^{\prime}$ for $t \leq 2 R_{1} /(\beta-\alpha)$, we have $\nu=$ $(\beta,-\mu) / \sqrt{1+\beta^{2}}$. We need

$$
\langle A(b)-A(c), \nu\rangle \geq 0 \text { for } b \leq c \leq b^{\prime} .
$$

The left-hand side has the same sign as $-(c-b) \beta+\langle f(c), \mu\rangle$ and this is nonnegative for $b \leq c \leq b^{\prime}$ by the definition of $\beta$. On the jump from $w=b^{\prime}$ to $w=a$, we take $\nu$ in the direction of $(-\alpha, \mu)$. It is required that $\langle A(a)-A(c), \nu\rangle \geq 0$ for $a \leq c \leq b^{\prime}$. This is equivalent to

$$
\alpha(c-a)-\langle f(c), \mu\rangle \geq 0 \text { for } a \leq c \leq b^{\prime}
$$

which holds by (2.1) and the definition of $\alpha$ since $\alpha \geq\langle f(b), \mu\rangle /(b-a)=0$.

The entropy condition holds across the jump from $w=b$ to $w=a$ for $t>$ $2 R_{1} /(\beta-\alpha)$ since $\nu=(0, \mu)$ and thus the entropy condition $\left(\mathrm{E}^{\prime}\right)$ reduces to $\langle f(c), \mu\rangle \leq 0$ for $a \leq c \leq b$. By Remark 1.7, we have

$$
\int_{B_{r}} \max \{0, w-u\}(t, x) d x \leq \int_{B_{r+l t}} \max \{0, w-u\}(0, x) d x=0
$$

for any $t$ and $r \geq 0$ where $l=\left[\sum_{i=1}^{n}\left(\max _{|u| \leq\left|b^{\prime}\right|+\left|a^{\prime}\right|}\left|f_{i}^{\prime}(u)\right|\right)^{2}\right]^{1 / 2}$. We conclude that $u \leq w$ for all $t$ and a.e. $x \in \mathbf{R}^{n}$, and hence $u \leq b$ for $t \geq t^{*}$ and a.e. $x \in \mathbf{R}^{n}$. To show that $u \geq a$ when $t \geq t^{*}$ involves the construction of a subsolution in a similar way.

A consequence of Theorem 2.3 is the following result on the large-time behavior of $u$.

THEOREM 2.4. Suppose $u_{0}$ is admissible. There exists $R_{3}>0$ and a set $M \subset$ $\mathbf{R}^{n}$ such that if $v(x)=b \cdot \chi_{M}(x)+a \cdot \chi_{\mathbf{R}^{n}-M}(x)$, then

(i) $u(t, x)-v(x-k t) \rightarrow 0$ in $L^{1}\left(\mathbf{R}^{n}\right)$ as $t \rightarrow \infty$,

(ii) $u(t, x)=v(x-k t)$ for $t>0$ and $|x-k t|>R_{3}$, and

(iii) $M \cap\left\{x \in \mathbf{R}^{n}:|x|>R_{3}\right\}=\left\{x \in \mathbf{R}^{n}:\langle x, \mu\rangle<0\right.$ and $\left.|x|>R_{3}\right\}$.

To prove this, we will need the following lemma. 
LEMMA 2.5. Suppose $u_{0}$ is admissible. There exists $R_{2}>R_{1}$ such that $u(t, x)$ $=\varphi(x-k t)$ if $t \geq 0$ and $|x-k t| \geq R_{2}$.

PROOF. By the change of variables (2.2), it is sufficient to prove the lemma assuming $f(a)=f(b)=0$, so that $k=0$ and $f=\tilde{f}$. Since $\varphi$ is then a steady state solution of (1.1) and $u_{0}=\varphi$ for $|x| \geq R_{1}$, it follows from estimate (1.3) that for some $r_{0}>R_{1}$,

$$
u\left(t^{*}, x\right)=\varphi(x) \text { for }|x| \geq r_{0}
$$

where $t^{*}$ is as defined in Theorem 2.3. We will show that in fact there exists $r_{1}>r_{0}$ such that

$$
u(t, x)=\varphi(x) \text { for } t \geq t^{*},|x| \geq r_{1} .
$$

To prove this we use the nondegeneracy condition, (2.1). It ensures that for some small $\delta>0,\langle f(c), \nu\rangle \leq 0$ for $a \leq c \leq b$ and all $\nu \in \mathbf{R}^{n}$ such that $A(\mu, \nu)<\delta$, where $A(\mu, \nu)$ is the angle between $\mu$ and $\nu$ that is not greater than $\pi$. Hence we can construct open sets, $M_{1}$ and $M_{2}$ (with smooth boundaries) and choose $r_{1}>r_{0}$ such that

$$
\begin{aligned}
& M_{1} \subset\{x:\langle x, \mu\rangle<0\} \subset M_{2}, \\
& B_{r_{0}} \equiv B_{r_{0}}(0) \subset M_{2}-M_{1}, \\
& M_{i} \cap\left\{x:|x| \geq r_{1}\right\}=\left\{x:\langle x, \mu\rangle<0 \text { and }|x| \geq r_{1}\right\} \quad \text { for } i=1,2, \text { and } \\
& \langle f(c), \nu\rangle \leq 0 \text { for } a \leq c \leq b,
\end{aligned}
$$

where $\nu$ is any outward pointing normal to $M_{1}$ or $M_{2}$. As a result the functions, $v_{i}(x)=b \cdot \chi_{M_{i}}(x)+a \cdot \chi_{\mathbf{R}^{n}-M_{i}}(x)$, are steady state solutions of (1.1). Since $v_{1}(x) \leq u\left(t^{*}, x\right) \leq v_{2}(x)$ for all $x$, we have

$$
v_{1}(x) \leq u(t, x) \leq v_{2}(x) \text { for } x \in \mathbf{R}^{n}, t \geq t^{*} .
$$

We conclude that

$$
u(t, x)=\varphi(x) \text { for }|x| \geq r_{1}, t \geq t^{*} .
$$

By (1.3) the above equation holds when $t \geq 0$ and $|x| \geq R_{2} \equiv r_{1}+l t^{*}$ which proves the conclusion of the lemma.

PROOF OF THEOREM 2.4. As before, we may assume that $f(a)=f(b)=0$ and hence $k=0$.

Suppose $u_{0} \in B V\left(\mathbf{R}^{n}\right)$. Consider $u_{h}(t, x)=u\left(t, x+h e_{i}\right)$ for any $i=1, \ldots, n$ and $|h| \leq R_{1}$, where $\left\{e_{1}, \ldots, e_{n}\right\}$ is the standard basis for $\mathbf{R}^{n}$. Since $u_{h}$ is a solution of (1.1), we have from Vol'pert's results (or Theorem 1.6) that $\operatorname{div} S\left(u_{h}, u\right) \leq 0$. Let $R=3 \cdot R_{2}$ with $R_{2}$ as defined in Lemma 2.5 and $B_{R}=B_{R}(0)$. By applying Green's theorem in the cylinder, $(0, t) \times B_{R}$, we obtain

$$
\begin{aligned}
\int_{B_{R}}\left|u\left(t, x+h e_{i}\right)-u(t, x)\right| d x & \leq \int_{B_{R}}\left|u\left(0, x+h e_{i}\right)-u(0, x)\right| d x \\
& -\int_{0}^{t} \int_{\partial B_{R}}\left\langle\frac{x}{R},\left(\left[f\left(u_{h}\right)-f(u)\right] \cdot \operatorname{sign}\left(u_{h}-u\right)\right)\right\rangle d \varkappa^{n} .
\end{aligned}
$$

By Lemma 2.5, $f\left(u_{h}\right)=f(u)=0$ on $\partial B_{R}$ for any $t$. Hence the third term is zero and

$$
\int_{B_{R}}\left|\frac{\partial u}{\partial x_{i}}(t, x)\right| d x \leq \int_{B_{R}}\left|\frac{\partial u}{\partial x_{i}}(0, x)\right| d x .
$$


This and equation (1.1) imply that

$$
\int_{-1}^{1} \int_{B_{R}}|(D u)(s+t, x)| d x d s \leq c \cdot \int_{B_{R}}\left|\left(\nabla_{x} u\right)(0, x)\right| d x
$$

for any $t>1$, where $D=\left(\partial / \partial t, \partial / \partial x_{1}, \ldots, \partial / \partial x_{n}\right)$.

Now multiply equation (1.1) by $\langle x, \mu\rangle$ and integrate over the cylinder $\left(t^{*}, T\right) \times B_{R}$. We obtain

$$
\begin{aligned}
& \int_{B_{R}} {[u(T, x)-u(0, x)] \cdot\langle x, \mu\rangle d x } \\
& \quad=\int_{t^{*}}^{T} \int_{B_{R}}\langle f(u), \mu\rangle d x d t-\int_{\partial B_{R}}\left\langle f(u), \frac{x}{R}\right\rangle \cdot\langle x, \mu\rangle d \not^{n} .
\end{aligned}
$$

The first term is bounded uniformly in $T$ (by estimate (1.3)) and the last term is zero. Since $a \leq u \leq b$ for $t \geq t^{*}$, we conclude using (2.1) that

$$
\int_{0}^{\infty} \int_{B_{R}}|(u-a)(u-b)| d x d t<\infty
$$

By Lemma 2.5, this can be restated as

$$
\int_{\mathbf{R}_{+}^{n+1}}|(u-a)(u-b)| d x d t<\infty .
$$

From the above estimates (and Lemma 2.5) we see that for some sequence, $t_{j} \rightarrow+\infty,\left\{u\left(s+t_{j}, x\right)\right\}$ converges in $L_{\text {loc }}^{1}\left((-1,1) \times \mathbf{R}^{n}\right)$ to a function, $v(s, x) \in$ $B V\left((-1,1) \times \mathbf{R}^{n}\right)$. The $L^{1}$ bound on $(u-a)(u-b)$ implies that $(v-a)(v-b)=0$ pointwise almost everywhere in $(-1,1) \times \mathbf{R}^{n}$. Thus

$$
v(s, x)=b \cdot \chi_{M}(s, x)+a \cdot \chi_{\mathbf{R}^{n}-M}(s, x)
$$

with $\chi_{M} \in B V\left((-1,1) \times \mathbf{R}^{n}\right)$. On the other hand, $\operatorname{div} S(v, c) \leq 0$ as a measure on $(-1,1) \times \mathbf{R}^{n}$ for all $c \in \mathbf{R}$. Hence $v$ is a solution of $(1.1)$ on $(-1,1) \times \mathbf{R}^{n}$. (See $[8$, $\S 16]$.) Since $f(a)=f(b)=0$, we have

$$
0=\partial v / \partial s+\operatorname{div} f(v)=\partial v / \partial s \quad \text { on }(-1,1) \times \mathbf{R}^{n} .
$$

Thus $\chi_{M}(s, x)=\chi_{M}(x)$.

Finally we show that $u(t, x)-v(x) \rightarrow 0$ in $L^{1}\left(\mathbf{R}^{n}\right)$ as $t \rightarrow \infty$. By Lemma 2.5 it is sufficient to prove convergence in $L^{1}\left(B_{R}\right)$. This follows from estimate (1.3) which implies that

$$
\int_{B_{R}(0)}|v(x)-u(t, x)| d x \leq \int_{B_{R}}\left|v(x)-u\left(s+t_{j}, x\right)\right| d x
$$

for $t \geq s+t_{j}$. This proves (i) when $u_{0} \in B V\left(\mathbf{R}^{n}\right)$; (ii) and (iii) follow from (i) and Lemma 2.5 with $R_{3}=R=3 \cdot R_{2}$.

For the case when $u_{0} \notin B V\left(\mathbf{R}^{n}\right)$ we can approximate $u_{0}$ by admissible functions, $u_{j}$, in $B V\left(\mathbf{R}^{n}\right)$ such that $u_{j} \rightarrow u_{0}$ in $L^{1}\left(B_{R_{3}}\right)$ and $u_{j}(x)=\varphi(x)$ for $|x| \geq R_{1}$. By Lemma 2.5 if $u_{j}(t, x)$ is the solution of (1.1) with initial values, $u_{j}(x)$, then

$$
u_{j}(t, x)=u(t, x)=\varphi(x) \text { for }|x| \geq R_{2} .
$$


By estimate (1.3) it follows that

$$
\int_{B_{R_{3}}}\left|v_{i}(x)-v_{j}(x)\right| d x \leq \int_{B_{R_{3}}}\left|u_{i}(x)-u_{j}(x)\right| d x
$$

where $v_{j}(x)=\lim _{t \rightarrow \infty} u_{j}(t, x)$. Hence for some set $M, \chi_{M_{j}}-\chi_{M} \rightarrow 0$ in $L^{1}\left(\mathbf{R}^{n}\right)$ and so

$$
u(t, x)-b \cdot \chi_{M}(x)-a \cdot \chi_{\mathbf{R}^{n}-M}(x) \rightarrow 0
$$

in $L^{1}\left(\mathbf{R}^{n}\right)$ as $t \rightarrow \infty$.

3. The $n$-dimensional case. We now get a more precise description of the set $M$ and the convergence of $u$ to $v(x-k t)$ in the special case

$$
n=d \equiv \operatorname{dim} \operatorname{span}\{\tilde{f}(c): a \leq x \leq b\} .
$$

THEOREM 3.2. There is a system of coordinates, $\left(\hat{y}, y_{n}\right)$, obtained by a rotation and a constant $C_{0}$ (both depending only on $\tilde{f}$ ) so that if $u_{0}$ is admissible then $M=\left\{\left(\hat{y}, y_{n}\right): y_{n}<g(\hat{y})\right\}$. The shock front, $g$, is Lipschitz continuous with Lipschitz constant at most $C_{0}$.

Proof. First assume $u_{0} \in B V\left(\mathbf{R}^{n}\right)$ and w.l.o.g. $f(a)=f(b)=0$ so that $f=\tilde{f}$. From the proof of Theorem $2.4, v(x)$ is a steady state solution of $(1.1)$ and $v \in B V\left(\mathbf{R}^{n}\right)$. Hence by $\left(\mathrm{E}^{\prime}\right)\langle f(c), \nu(x)\rangle \leq 0$ for $a \leq c \leq b$ and $\not^{n-1}$ a.e. $x \in \Gamma(v)$ where $\nu(x)$ is the normal to $\Gamma(v)$ at $x$. Consider the convex set

$$
\Omega=\left\{\sum_{i=1}^{m} a_{i} f\left(c_{i}\right): a_{i} \geq 0, a \leq c_{i} \leq b, m<\infty\right\} .
$$

We have $\nu(x) \in \bigcap_{z \in \Omega}\left\{w \in \mathbf{R}^{n}:\langle w, z\rangle \leq 0\right\}$ for each such $x \in \Gamma(v)$. From (3.1) the interior of $\Omega$ is nonempty. Choose $\alpha \in \Omega^{0}$ with $|\alpha|=1$ and $\delta>0$ so that

$$
E \equiv\left\{z \in \mathbf{R}^{n}: A(z, \alpha) \leq \delta\right\} \subset \Omega \text {. }
$$

Then

$$
\nu(x) \in \bigcap_{z \in E}\left\{w \in \mathbf{R}^{n}:\langle w, z\rangle \leq 0\right\}=\left\{w \in \mathbf{R}^{n}: A(-\alpha, w) \leq \pi / 2-\delta\right\} .
$$

It follows from the theory of sets with locally finite perimeter [3, Theorem 4.8] that in a rotated system of coordinates (with $e_{n}=-\alpha$ ) there exists a function $g(\hat{y})$ such that $M=\left\{\left(\hat{y}, y_{n}\right): y_{n}<g(\hat{y})\right\}$ where $v=b \cdot \chi_{M}+a \cdot \chi_{\mathbf{R}^{n}-M}$ and

$$
\left|g\left(\hat{y}_{1}\right)-g\left(\hat{y}_{2}\right)\right| \leq \tan (\pi / 2-\delta) \cdot\left|\hat{y}_{1}-\hat{y}_{2}\right| \equiv C_{0} \cdot\left|\hat{y}_{1}-\hat{y}_{2}\right| \text {. }
$$

If $u_{0} \notin B V\left(\mathbf{R}^{n}\right)$ we take a sequence, $\left\{u_{j}(0, x)\right\}$, of admissible data such that $u_{j}(0, x) \in B V\left(\mathbf{R}^{n}\right)$ and $u_{j}(0, x) \rightarrow u_{0}(x)$ in $L_{\text {loc }}^{1}\left(\mathbf{R}^{n}\right)$ as $j \rightarrow \infty$. We have seen that the corresponding steady state limits satisfy $v_{j} \rightarrow v$ in $L_{\text {loc }}^{1}\left(\mathbf{R}^{n}\right)$ as $j \rightarrow \infty$. From the above argument we have $M_{j}=\left\{\left(\hat{y}, y_{n}\right): y_{n}<g_{j}(\hat{y})\right\}$ where the $g_{j}$ are uniformly Lipschitz continuous and

$$
\left\langle f(c),\left(-\nabla g_{j}(\hat{y}), 1\right)\right\rangle \leq 0 \text { for } a \leq c \leq b
$$

and $L^{n-1}$ a.e. $\hat{y}$. (Here it is understood that $f(c)$ is expressed in the new coordinates, $\left.\left(\hat{y}, y_{n}\right).\right)$ It follows that $M=\left\{\left(\hat{y}, y_{n}\right): y_{n}<g(\hat{y})\right\}$ for some Lipschitz continuous $g$ satisfying the same conditions.

The uniform bound on the Lipschitz constant of the shock front yields the following stability result. 
THEOREM 3.3. Let $u_{1}(0, x), u_{2}(0, x)$ be admissible data and $v_{1}(x-k t)$, $v_{2}(x-k t)$ be the asymptotic limits with $g_{1}, g_{2}$ the corresponding shock fronts. There is a constant $C_{1}(\tilde{f})<\infty$ so that

$$
\left\|g_{1}-g_{2}\right\|_{L^{\infty}\left(\mathbf{R}^{n-1}\right)} \leq C_{1}\left(\int_{B_{R_{1}}}\left|u_{1}(0, x)-u_{2}(0, x)\right| d x\right)^{1 / n} .
$$

ProOF. Assume that $f(a)=f(b)=0$. In the coordinates $\left(\hat{y}, y_{n}\right)$ we have $M_{i}=$ $\left\{\left(\hat{y}, y_{n}\right): y_{n}<g_{i}(\hat{y})\right\}$ and $\left|\nabla g_{i}\right| \leq C_{0}$ for $i=1,2$ where $v_{i}=b \cdot \chi_{M_{i}}+a \cdot \chi_{\mathbf{R}^{n}-M_{i}}$. As in the proof of Theorem 2.4, Green's theorem implies that

$$
\begin{aligned}
(b-a) \cdot \int_{\mathbf{R}^{n-1}}\left|g_{1}-g_{2}\right| d \hat{y} & =\int_{B_{R_{3}}}\left|v_{1}-v_{2}\right| d x \\
& \leq \int_{B_{R_{1}}}\left|u_{1}(0, x)-u_{2}(0, x)\right| d x .
\end{aligned}
$$

But since $\left|\nabla g_{1}\right|$ and $\left|\nabla g_{2}\right|$ are a priori bounded, we conclude that if $\mid g_{1}\left(\hat{y}_{0}\right)-$ $g_{2}\left(\hat{y}_{0}\right) \mid=\delta>0$ then $\left|g_{1}(\hat{y})-g_{2}(\hat{y})\right| \geq \delta / 2$ for $\hat{y} \in B_{\delta / 4 C_{0}}\left(\hat{y}_{0}\right)$. Thus

$$
\left(\left\|g_{1}-g_{2}\right\|_{L^{\infty}\left(\mathbf{R}^{n-1}\right)}\right)^{n} \leq C_{1}(\tilde{f}) \cdot \int_{\mathbf{R}^{n-1}}\left|g_{1}-g_{2}\right| d \hat{y} .
$$

REMARK 3.4. We will need to apply a version of this theorem when $u_{1}(0, x)$ and $u_{2}(0, x)$ are admissible relative to shocks that differ by a small translation, i.e. $u_{1}(0, x)=\varphi(x)$ and $u_{2}(0, x)=\varphi(x+\eta \mu)$ for $|x| \geq R_{1}$. If we assume w.l.o.g. that $C_{0}>1$ and take $R_{4}=5 C_{0} \cdot \max \left\{R_{3}, C_{0} \eta\right\}$ then the same argument yields

$$
\left\|g_{1}-g_{2}\right\|_{L^{\infty}\left(\mathbf{R}^{n-1}\right)} \leq C_{1}\left(\int_{B_{R_{4}}}\left|u_{1}(0, x)-u_{2}(0, x)\right| d x\right)^{1 / n} .
$$

DEFINITION 3.5. A function $h(x)$ is piecewise continuous iff there exists a finite number of mutually disjoint domains, $\left\{D_{i}\right\}_{i=1}^{k}$, such that $\left.h\right|_{D_{i}}$ has a continuous extension to $D_{i} \bigcup \partial D_{i}$ and $L^{n}\left(\mathbf{R}^{n}-\bigcup_{i=1}^{k} D_{i}\right)=0$.

We will prove that if $u_{0}$ is admissible, piecewise continuous, and dist $(x-k t, \partial M)$ $>\varepsilon>0$, then $u(t, x)=v(x-k t)$ after a finite time depending on $\varepsilon$ and $\tilde{f}$. (See Theorem 3.12.) It will be convenient to use the following notation.

DEFINITION 3.6. If

$$
w(t, x) \in C\left([0, \infty) ; L_{\mathrm{loc}}^{1}\left(\mathbf{R}^{n}\right)\right)
$$

we denote by $U(w)(t, x)(L(w)(t, x))$ the upper (lower) Lebesgue limit in $x$ :

$$
U(w)(t, x)=\varlimsup_{r \rightarrow 0} f_{B_{r}(x)} w(t, z) d z, \quad L(w)(t, x)=\varliminf_{r \rightarrow 0} f_{B_{r}(x)} w(t, z) d z .
$$

Note that for each $t>0, U(w)(t, x)=L(w)(t, x)=w(t, x)$ for a.e. $x$.

The following lemma implies the uniform convergence of $u(t, x)$ away from the shock front. 
LEMMA 3.7. Suppose that $u_{0}$ is admissible, piecewise continuous, and $g(\hat{y})$ is the shock front for the asymptotic limit. Then given $\varepsilon, \sigma>0$ there is a constant $T(\varepsilon, \sigma)<\infty$ so that in the coordinates $\left(\hat{y}, y_{n}\right)$ for $t>T$ we have

(1) $a \leq u(t, y) \leq a+\sigma$ for a.e. $y$ with $y_{n}-k_{n} t \geq g(\hat{y}-\hat{k} t)+\varepsilon$,

(2) $b \geq u(t, y) \geq b-\sigma$ for a.e. $y$ with $y_{n}-k_{n} t \leq g(\hat{y}-\hat{k} t)-\varepsilon$, where $\left(\hat{k}, k_{n}\right)$ is the representation of $k=[f(b)-f(a)] /(b-a)$ in the coordinates, $\left(\hat{y}, y_{n}\right)$.

ProOF. Assume $f(a)=f(b)=0$ so that $k=0$. Note that from Lemma 2.3 the first inequalities in (1) and (2) hold for $t \geq t^{*}$.

For any $\delta \in(0,1)$ we can find functions $q_{\delta}(x), p_{\delta}(x)$ and a positive constant, $\tau(\delta) \leq \delta$, so that the following three properties hold. First,

$$
\text { ess inf } u_{0} \leq q_{\delta}(x) \leq u_{0}(x+h) \leq p_{\delta}(x) \leq \text { ess } \sup u_{0}
$$

for any $h \in \mathbf{R}^{n}$ with $|h| \leq \tau$. Second, if $q_{\delta}(t, x)$ and $p_{\delta}(t, x)$ are the solutions of (1.1) with initial values $p_{\delta}(x)$ and $q_{\delta}(x)$, we have $q_{\delta}(t, x)=\varphi(x+\tau \mu)$ and $p_{\delta}(t, x)=\varphi(x-\tau \mu)$ for $|x| \geq R, t \geq 0$, where $R$ is independent of $\delta$. Third,

$$
\int_{B_{R}}\left(p_{\delta}-q_{\delta}\right) d x \leq \delta
$$

If (1) does not hold there exists a sequence of points $\left(t^{m}, y^{m}\right)$ in the rotated coordinates, $y=\left(\hat{y}, y_{n}\right)$, with $t^{m} \rightarrow \infty$ and $y^{m} \rightarrow y^{0}$ as $m \rightarrow \infty$ so that

$$
U(u)\left(t^{m}, y^{m}\right)>a+\sigma \text { and } y_{n}^{m} \geq g\left(\hat{y}^{m}\right)+\varepsilon .
$$

For $\delta$ fixed and any $\bar{y} \in B_{\tau / 2}\left(y^{0}\right)$ it follows from Remark 1.7 that

$$
u\left(t^{m}, y+h\right) \leq p_{\delta}\left(t^{m}, y\right) \text { for a.e. } y
$$

where $h=y^{m}-\bar{y}, m$ is sufficiently large and $p_{\delta}(t, y)$ denotes the function $p_{\delta}(t, x)$ expressed in the rotated spacial coordinates. Thus

$$
U(u)\left(t^{m}, y^{m}\right) \leq U\left(p_{\delta}\right)\left(t^{m}, \bar{y}\right) .
$$

As a result,

$$
a+\sigma \leq p_{\delta}\left(t^{m}, \bar{y}\right) \quad \text { for a.e. } \bar{y} \in B_{\tau / 2}\left(y^{0}\right)
$$

if $m$ is large. Let $v_{\delta}(x)$ be the asymptotic limit of $p_{\delta}(t, x)$,

$$
v_{\delta}(x)=b \cdot \chi_{M_{\delta}}(x)+a \cdot \chi_{\mathbf{R}^{n}-M_{\delta}}(x) .
$$

It follows from (3) and Theorem 2.4 that $B_{\tau / 2}\left(y^{0}\right) \subset M_{\delta}$. Using $g_{\delta}(\hat{y})$ to denote the shock front for $v_{\delta}$ we get $\varepsilon \leq\left|g_{\delta}\left(\hat{y}^{0}\right)-g\left(\hat{y}^{0}\right)\right|$. But from (3.4)

$$
\left\|g-g_{\delta}\right\|_{L^{\infty}\left(\mathbf{R}^{n-1}\right)} \leq C\left(\int_{B_{R_{4}}}\left|u_{0}(x)-p_{\delta}(0, x)\right| d x\right)^{1 / n} \leq C \cdot \delta^{1 / n} .
$$

Since $\delta$ can be chosen arbitrarily small this is a contradiction. The verification of (2) is a similar argument using $q_{\delta}(x)$.

We shall improve on this estimate by using super and subsolutions. The construction of the supersolution is done below in detail. 
LEMMA 3.8. Let $f(c)$ be expressed in the coordinates $\left(\hat{y}, y_{n}\right)$ and suppose $f(a)=$ $f(b)=0$. Let $g(\hat{y})$ be any Lipschitz continuous function so that for almost every $\hat{y}$,

$$
\begin{array}{ll}
\frac{\langle(\nabla g,-1), f(c)\rangle}{c-a} \geq m>0 & \text { for } a \leq c \leq \frac{a+b}{2} \\
\langle(\nabla g,-1), f(c)\rangle \geq 0 & \text { for } a \leq c \leq b .
\end{array}
$$

Suppose $\eta$ and $\delta$ are positive constants. Define

$$
w\left(t, \hat{y}, y_{n}\right)= \begin{cases}b & \text { for } y_{n} \leq g(\hat{y})-\delta+\eta t \\ (a+b) / 2 & \text { for } g(\hat{y})-\delta+\eta t \leq y_{n} \leq g(\hat{y})-m t \\ a & \text { for } g(\hat{y})-m t \leq y_{n}\end{cases}
$$

when $0 \leq t \leq \delta /(m+\eta)$

$$
w\left(t, \hat{y}, y_{n}\right)= \begin{cases}b & \text { for } y_{n}<g(\hat{y})-m \delta /(m+\eta) \\ a & \text { for } y_{n} \geq g(\hat{y})-m \delta /(m+\eta)\end{cases}
$$

when $t \geq \delta /(m+\eta)$.

Then $w$ is a supersolution of (1.1) provided $\eta$ is sufficiently large depending only on $f$ and the Lipschitz constant of $g$.

PROOF. Since $w$ is piecewise constant,

$$
\partial_{t} w+\operatorname{div}_{x} f(w)=\left.\left\langle\mid\left[\left(w^{+}, f\left(w^{+}\right)\right)-\left(w^{-}, f\left(w^{-}\right)\right)\right], \nu(t, y)\right\rangle d \nvdash^{n}\right|_{\Gamma(w)} .
$$

We must verify that

$$
\left\langle\left[\left(w^{+}, f\left(w^{+}\right)\right)-(c, f(c))\right], \nu\right\rangle \geq 0 \text { for } w^{+} \leq c \leq w^{-}
$$

and $\nvdash^{n}$ a.e. $(t, y) \in \Gamma(w)$. The jump between the states $b$ and $(a+b) / 2$ is determined by

$$
0=y_{n}-g(\hat{y})+\delta-\eta t
$$

We have

$$
\nu=(-\eta,-\nabla g, 1) / \sqrt{\eta^{2}+|\nabla g|^{2}+1}
$$

Thus we need to establish that

$$
-\left(\frac{a+b}{2}-c\right) \eta+\left\langle\left[f\left(\frac{a+b}{2}\right)-f(c)\right],(-\nabla g, 1)\right\rangle \geq 0 \text { for } \frac{a+b}{2} \leq c \leq b .
$$

This is equivalent to

$$
\eta \geq\left\langle\left[f\left(\frac{a+b}{2}\right)-f(c)\right],(-\nabla g, 1)\right\rangle /\left(\frac{a+b}{2}-c\right)
$$

which is valid for $\eta$ sufficiently large. For the jump between $(a+b) / 2$ and $a$ we have $0=y_{n}-g(\hat{y})+m t, \nu=(m,-\nabla g, 1) / \sqrt{m^{2}+|\nabla g|^{2}+1}$. We must verify that

$$
m(a-c)-\langle f(c),(-\nabla g, 1)\rangle \geq 0 \text { for } a \leq c \leq(a+b) / 2 \text {. }
$$

This is equivalent to the first inequality of (3.9).

On the jump between $b$ and $a$ for $t \geq \delta /(m+\eta)$ the entropy condition is equivalent to the second inequality of (3.9). This completes the proof.

The shock front $g$ satisfies

$$
g(\hat{y})=\langle l, \hat{y}\rangle \quad \text { for }|\hat{y}| \geq R_{3}
$$


where $(l,-1) / \sqrt{|l|^{2}+1}$ is the representation of $-\mu$ in the coordinates $\left(\hat{y}, y_{n}\right)$. Hence

$$
\langle f(c),(l,-1)\rangle \geq \theta(c-a)(b-c) \sqrt{|l|^{2}+1} \text { for } a \leq c \leq b .
$$

In general the first inequality of (3.9) does not hold. However, $g$ can be uniformly approximated from above and below by functions, $g_{1, \varepsilon}$ and $g_{2, \varepsilon}$ respectively, where

$$
g_{j, \varepsilon}(\hat{y})=g((1-\varepsilon) \hat{y})+\varepsilon\langle l, \hat{y}\rangle-(-1)^{j} \cdot c \varepsilon
$$

for $0<\varepsilon<\frac{1}{2}, j=1,2$, and $c>0$ is sufficiently large (independent of $\varepsilon$ ). We have

$$
\nabla g_{j, \varepsilon}(\hat{y})=(1-\varepsilon) \cdot \nabla g((1-\varepsilon) \hat{y})+\varepsilon l
$$

so that

$$
\begin{aligned}
\langle f(c), & \left.\left(\nabla g_{j, \varepsilon}(\hat{y}),-1\right)\right\rangle \\
= & (1-\varepsilon)\langle f(c),(\nabla g((1-\varepsilon) \hat{y}),-1)\rangle+\varepsilon\langle f(c),(l,-1)\rangle \\
\geq & \varepsilon \theta \sqrt{|l|^{2}+1} \cdot(c-a)(b-c) \text { for } a \leq c \leq b .
\end{aligned}
$$

Thus supersolutions can be constructed with $g_{1, \varepsilon}$.

Similarly one can construct subsolutions with $g_{2, \varepsilon}$. The inequalities analogous to (3.9) which we require are

$$
\begin{aligned}
& \frac{\langle(\nabla g,-1), f(c)\rangle}{b-c} \geq \eta>0 \text { for } \frac{a+b}{2} \leq c \leq b, \\
& \langle(\nabla g,-1), f(c)\rangle \geq 0 \text { for } a \leq c \leq b .
\end{aligned}
$$

These hold with $g$ replaced by $g_{2, \varepsilon}$ and $\eta$ sufficiently small by inequality (3.10). Thus the formula of Lemma 3.8 defines a subsolution if $m$ is sufficiently large.

We now improve on Lemma 3.7 in the sense that we show $u(t, x)=v(x-k t)$ outside of an arbitrarily small neighborhood of the shock front after finite time.

THEOREM 3.11. Let $u(t, x)$ be a solution of (1.1) with $u_{0}(x)$ admissible and piecewise continuous. Then in the coordinates $\left(\hat{y}, y_{n}\right)$, given $\delta>0$ there is a constant $T(\delta)<\infty$ so that

$$
\begin{aligned}
& u(t, y)=a \text { for a.e. } y \text { with } y_{n}-k_{n} t \geq g(\hat{y}-\hat{k} t)+\delta, t \geq T \\
& u(t, y)=b \text { for a.e. } y \text { with } y_{n}-k_{n} t \leq g(\hat{y}-\hat{k} t)-\delta, t \geq T
\end{aligned}
$$

ProOF. Assume that $f(a)=f(b)=0$. Suppose there is a sequence $\left(t^{j}, y^{j}\right)$ with $y^{j} \rightarrow y^{0}, t^{j} \rightarrow \infty$ as $j \rightarrow \infty$ such that $U(u)\left(t^{j}, y^{j}\right)>a$ and $y_{n}^{0} \geq g\left(\hat{y}^{0}\right)+\delta$. Suppose $\varepsilon \ll \delta$ and set

$$
h_{\tau}(\hat{y})=g_{1, \varepsilon}(\hat{y})+\tau, \quad M_{\tau}=\left\{\left(\hat{y}, y_{n}\right): y_{n}<h_{\tau}(\hat{y})\right\}
$$

Define

$$
\tau(t)=\inf \left\{\tau:\{y: U(u)(t, y)>a\} \subset M_{\tau}\right\}
$$

This is well defined for each $t>0$ by Theorem 2.4. Set $v_{\tau}=b \cdot \chi_{M_{\tau}}+a \cdot \chi_{\mathbf{R}^{n}-M_{\tau}}$. By (3.10), $v_{\tau}$ is a steady state solution of (1.1). From Remark 1.7 and Theorem 2.3 we get $v_{\tau(t)}(y) \geq u(s, y)$ for $s \geq t$ and a.e. $y$. Thus $\tau(t)$ is nonincreasing as $t \uparrow \infty$.

Set $\tau_{0}=\lim _{t \rightarrow \infty} \tau(t)$. If $\varepsilon$ is sufficiently small $y_{n}^{0} \geq h_{3 \delta / 4}\left(\hat{y}^{0}\right)$, so $\tau_{0} \geq 3 \delta / 4$. Hence $h_{\tau_{0}} \geq g+3 \delta / 4$. Choose $T$ so large that $\tau(T) \leq \varepsilon^{2}+\tau_{0}$; using Lemma 3.7, assume $T$ is large enough to ensure that

$$
U(u)(t, y) \leq \frac{a+b}{2} \text { for } y_{n} \geq g(\hat{y})+\frac{\delta}{2}, t \geq T
$$


We can use the supersolution from the previous lemma for $t \geq T$ with $g$ replaced by $h_{\tau(T)}, \delta$ replaced by $\delta / 4, m=\varepsilon \theta(b-a)\left(\sqrt{|l|^{2}+1}\right) / 2 \equiv c_{1} \varepsilon$, and

$$
w(T, y)= \begin{cases}b & \text { for } y_{n} \leq h_{\tau(T)}(\hat{y})-\delta / 4, \\ (a+b) / 2 & \text { for } h_{\tau(T)}(\hat{y})-\delta / 4 \leq y_{n} \leq h_{\tau(T)}(\hat{y}), \\ a & \text { for } h_{\tau(T)}(\hat{y})<y_{n} .\end{cases}
$$

It follows that for $t \geq T+\delta / 4\left(\eta+c_{1} \varepsilon\right) \equiv T+c_{2} \delta, U(u)(t, y) \equiv a$ on the set $\left\{y: y_{n}>h_{\tau(T)}(\hat{y})-c_{1} c_{2} \delta \varepsilon\right\}$. Thus $\tau_{0} \leq \tau(T)-c_{1} c_{2} \delta \varepsilon$ which implies that $c_{1} c_{2} \delta \varepsilon \leq$ $\varepsilon^{2}$. This is a contradiction since $\varepsilon$ can be taken arbitrarily small. The proof of (1) is complete; (2) follows in a similar way using subsolutions.

An immediate consequence of this theorem is

THEOREM 3.12. Suppose $u_{0}$ is admissible, piecewise continuous, and $k=$ $[f(b)-f(a)] /(b-a)$. Then for any $\varepsilon>0$ there is a constant $T_{\varepsilon}<\infty$ such that

$$
u(t, x)=v(x-k t) \quad \text { for a.e. } x \in \mathbf{R}^{n},
$$

when $t>T_{\varepsilon}$ and $\operatorname{dist}(x-k t, \partial M)>\varepsilon$.

4. The lower dimensional case. We now analyze the situation when $d<n$. By an appropriate rotation of the spacial coordinates we can assume that

$$
f(c)=\left(h_{1}(c), \ldots, h_{d}(c), j_{1}(c), \ldots, j_{n-d}(c)\right),
$$

$\operatorname{dim} \operatorname{span}\{\tilde{h}(c): a \leq c \leq b\}=d$, and $\tilde{j}(c)=0$ for $a \leq c \leq b$, where

$$
\begin{gathered}
\tilde{h}(c) \equiv h(c)-h(a)-(c-a)[h(b)-h(a)] /(b-a), \\
\tilde{j}(c) \equiv j(c)-j(a)-(c-a)[j(b)-j(a)] /(b-a) .
\end{gathered}
$$

Moreover the projection of $\mu$ on $\mathbf{R}^{d}$ is nonzero and if $\mu^{\prime}=P_{\mathbf{R}^{d}}(\mu) /\left\|P_{\mathbf{R}^{d}}(\mu)\right\|$ we have

$$
\left\langle\mu^{\prime}, \tilde{h}(c)\right\rangle \leq\langle\mu, \tilde{f}(c)\rangle \leq-\theta(c-a)(b-c) \text { for } a \leq c \leq b .
$$

We use the notation $x=\left(x^{\prime}, x^{\prime \prime}\right)$ where $x^{\prime} \in \mathbf{R}^{d}$ and $x^{\prime \prime} \in \mathbf{R}^{n-d}$.

LEMMA 4.1. Suppose $u_{0}$ is admissible and $k=\left(k^{\prime}, k^{\prime \prime}\right)=[f(b)-f(a)] /(b-a)$. Then for $t \geq T$ (where $T$ is such that $a \leq u(t, x) \leq b$ for $t \geq T$ ) we have $u(t, x)=$ $\tilde{u}\left(t, x^{\prime}-k^{\prime} t ; x^{\prime \prime}-k^{\prime \prime} t\right)$, where $\tilde{u}\left(t, x^{\prime} ; x^{\prime \prime}\right) \in C\left([T, \infty) ; L_{\text {loc }}^{1}\left(\mathbf{R}^{d}\right)\right)$ for $L^{n-d}$ a.e. $x^{\prime \prime}$ and is the solution of the Cauchy problem

$$
\left\{\begin{array}{l}
\partial_{t} w+\operatorname{div}_{x^{\prime}} \tilde{h}(w)=0 \text { for } t \geq T, x^{\prime} \in \mathbf{R}^{d}, \\
w\left(T, x^{\prime}\right)=u\left(T, x^{\prime}+k^{\prime} T ; x^{\prime \prime}+k^{\prime \prime} T\right) .
\end{array}\right.
$$

Moreover for each such $x^{\prime \prime}, u\left(T, x^{\prime}+k^{\prime} T ; x^{\prime \prime}+k^{\prime \prime} T\right)$ is admissible relative to a nondegenerate shock of the form

$$
\varphi\left(x^{\prime} ; x^{\prime \prime}\right)= \begin{cases}a & \text { for }\left\langle x^{\prime}-x_{0}^{\prime}\left(x^{\prime \prime}\right), \mu^{\prime}\right\rangle>0 \\ b & \text { for }\left\langle x^{\prime}-x_{0}^{\prime}\left(x^{\prime \prime}\right), \mu^{\prime}\right\rangle<0 .\end{cases}
$$

ProOF. As in $\S 2$ we assume that $f(a)=f(b)=0$ so that $\tilde{h}(c)=h(c), j(c)=0$ for $a \leq c \leq b$, and $k=0$.

It suffices to prove the lemma in the region $\Omega_{N}=\{(t, x): T \leq t<T+N,|x| \leq N\}$ for any $N<\infty$. From (1.3) we see that $u$ is uniquely determined on $\Omega_{N}$ by $u(T, x)$ 
with $|x| \leq N(1+l)$. Thus without loss of generality we can assume that the solution is redefined at time $t=T$ for $|x|>N(1+l)$ so that $u(T, x) \equiv a$ for $|x| \geq 2 N(1+l)$.

To begin with suppose $u(T, x) \in C^{\infty}\left(\mathbf{R}^{n}\right)$ and $h \in C^{\infty}\left(\mathbf{R}^{1} ; \mathbf{R}^{d}\right)$. For $\varepsilon>0$ let $v_{\varepsilon}\left(t, x^{\prime} ; x^{\prime \prime}\right)$ be the solution to

$$
\left\{\begin{array}{l}
\partial_{t} v_{\varepsilon}+\operatorname{div}_{x^{\prime}} h\left(v_{\varepsilon}\right)=\varepsilon \Delta_{x^{\prime}} v_{\varepsilon} \quad \text { for } t \geq T \text { and } x \in \mathbf{R}^{n} \\
v_{\varepsilon}\left(T, x^{\prime} ; x^{\prime \prime}\right)=u(T, x) \text { for } x \in \mathbf{R}^{n}
\end{array}\right.
$$

Thus we view $x^{\prime \prime}$ as a parameter in the initial conditions. The theory of parabolic equations implies that a unique solution exists with $a \leq v_{\varepsilon} \leq b$ and

$$
v_{\varepsilon} \in C^{\infty}\left([T, \infty) \times \mathbf{R}^{n}\right) .
$$

From $[8, \S \S 17.2$ and 18.1], we obtain the estimates

$$
\begin{aligned}
& \int_{\mathbf{R}^{d}}\left|\nabla_{x^{\prime}} v_{\varepsilon}\right|\left(t, x^{\prime} ; x^{\prime \prime}\right) d x^{\prime} \\
& \quad \leq \int_{\mathbf{R}^{d}}\left|\nabla_{x} v_{\varepsilon}\right|\left(T, x^{\prime} ; x^{\prime \prime}\right) d x^{\prime} \quad \text { for } t \geq T, x^{\prime \prime} \in \mathbf{R}^{n-d}, \\
& \int_{T}^{T^{\prime}} \int_{\mathbf{R}^{d}}\left|\partial_{t} v_{\varepsilon}\right|\left(t, x^{\prime} ; x^{\prime \prime}\right) d x^{\prime} d t \\
& \leq c\left(T^{\prime}, l\right) \cdot \int_{\mathbf{R}^{d}}\left(\left|\nabla_{x^{\prime}} v_{\varepsilon}\right|\left(T, x^{\prime} ; x^{\prime \prime}\right)+\varepsilon\left|\Delta_{x^{\prime}} v_{\varepsilon}\right|\left(T, x^{\prime} ; x^{\prime \prime}\right)\right) d x^{\prime}
\end{aligned}
$$

for $T \leq T^{\prime}<\infty$ and $x^{\prime \prime} \in \mathbf{R}^{n-d}$. As a result we get

$$
\begin{aligned}
& \int_{\mathbf{R}^{n}}\left|\nabla_{x} v_{\varepsilon}\right|(t, x) d x \leq \int_{\mathbf{R}^{n}}\left|\nabla_{x} v_{\varepsilon}\right|(T, x) d x \quad \text { for } t \geq T, \\
& \int_{T}^{T^{\prime}} \int_{\mathbf{R}^{n}}\left|\partial_{t} v_{\varepsilon}\right|(t, x) d x d t \\
& \quad \leq C\left(T^{\prime}, l\right) \cdot \int_{\mathbf{R}^{n}}\left(\left|\nabla_{x} v_{\varepsilon}\right|(T, x)+\varepsilon\left|\Delta_{x^{\prime}} v_{\varepsilon}\right|(T, x)\right) d x
\end{aligned}
$$

If we assume only that $h \in C^{1}\left([a, b] ; \mathbf{R}^{d}\right)$ then by taking a sequence of smooth functions, $h_{j}$, converging to $h$ in this space one finds that the corresponding solutions, $v_{\varepsilon, j}$, together with $\nabla_{x} v_{\varepsilon, j}$ converge uniformly in $\left[T, T^{\prime}\right] \times \mathbf{R}^{n}$. Also

$$
\sup _{\left[T, T^{\prime}\right] \times R^{n}}\left|\partial_{t} v_{\varepsilon, j}\right| \leq C<\infty
$$

independent of $j$. Letting $j \rightarrow \infty$ the limit, $v_{\varepsilon}$, will be a solution of (4.3); the integrands in (a)-(d) are all locally integrable functions and since the right-hand sides of the four inequalities are independent of $j$ they remain valid in the limit.

From (c) and (d) we see that a sequence $\varepsilon_{j} \downarrow 0$ can be chosen so that $v_{\varepsilon_{j}} \rightarrow w \in$ $B V\left((T, \infty) \times \mathbf{R}^{n}\right)$ with convergence in $L_{\text {loc }}^{1}\left((T, \infty) \times \mathbf{R}^{n}\right)$. We can further assume that there is a set, $Z$, of full $\mathcal{L}^{n-d}$ measure so that

$$
v_{\varepsilon_{j}}(t, x) \rightarrow w\left(t, x^{\prime} ; x^{\prime \prime}\right) \text { in } L_{\text {loc }}^{1}\left((T, \infty) \times \mathbf{R}^{d}\right)
$$

for $x^{\prime \prime} \in Z$. From (a) and (b) we see that $w\left(t, x^{\prime} ; x^{\prime \prime}\right) \in B V\left((T, \infty) \times \mathbf{R}^{d}\right)$ for each $x^{\prime \prime} \in Z$. Thus (as in $\S 1$ ) we can define

$$
\bar{w}\left(t, x^{\prime} ; x^{\prime \prime}\right) \equiv \lim _{r \rightarrow \infty} f_{B_{r}\left(\left(t, x^{\prime}\right)\right)} w\left(\tau, z ; x^{\prime \prime}\right) d \tau d z, \quad t>T, x^{\prime \prime} \in Z .
$$


From $[8, \S 18]$, for each such $x^{\prime \prime}, \bar{w}\left(t, x^{\prime} ; x^{\prime \prime}\right)$ is the solution to (4.2). By the same argument as in $[\mathbf{8}, \S 18], \bar{w}(t, x)$ (the Lebesgue limit in $\mathbf{R}^{n+1}$ ) is the solution to (1.1) with $\bar{w}(t, x) \rightarrow u(T, x)$ as $t \downarrow T$ in $L_{\text {loc }}^{1}\left(\mathbf{R}^{n}\right)$. We point out that $\bar{w}\left(t, x^{\prime} ; x^{\prime \prime}\right)$ agrees with $\bar{w}(t, x)$ as an element in $C\left([T, \infty) ; L_{\text {loc }}^{1}\left(\mathbf{R}^{n}\right)\right)$. We point out that $\bar{w}\left(t, x^{\prime} ; x^{\prime \prime}\right)$ agrees with $\bar{w}(t, x)$ as an element in $C\left([T, \infty) ; L_{\text {loc }}^{1}\left(\mathbf{R}^{n}\right)\right)$. Thus the proof is complete when $u(T, x) \in C^{\infty}\left(\mathbf{R}^{n}\right)$.

For the general case consider a sequence of functions $u_{j}(T, x) \in C^{\infty}\left(\mathbf{R}^{n}\right)$ with $a \leq u_{j} \leq b, u_{j}(T, x)=a$ for $|x| \geq 2 N(1+l)$, and $u_{j} \rightarrow u$ in $L_{\text {loc }}^{1}\left(\mathbf{R}^{n}\right)$. Using equation (1.3) we see that $\left\{\bar{u}_{j}\left(t, x^{\prime} ; x^{\prime \prime}\right)\right\}, j=1,2, \ldots$, forms a Cauchy sequence in $C\left([T, T+N] ; L_{\text {loc }}^{1}\left(\mathbf{R}^{n}\right)\right)$, and that for a.e. $x^{\prime \prime} \in \mathbf{R}^{n-d}$, the sequence is Cauchy in $C\left([T, T+N] ; L_{\text {loc }}^{1}\left(\mathbf{R}^{d}\right)\right)$. For a subsequence, $\bar{u}_{j}\left(t, x^{\prime} ; x^{\prime \prime}\right) \rightarrow u(t, x)$ for all $t \in$ $[T, T+N]$ and a.e. $x^{\prime \prime} \in \mathbf{R}^{n-d}$, pointwise almost everywhere in $\mathbf{R}^{d}$. This limit has the required properties.

With this result and those from $\S \S 2$ and 3 we have the following.

THEOREM 4.4. Let $u_{0}(x)$ be admissible and let $b \cdot \chi_{M}(x-k t)+a \cdot \chi_{\mathbf{R}^{n}-M}(x-k t)$ be the asymptotic limit of $u(t, x)$. Then for almost every $x_{0}^{\prime \prime} \in \mathbf{R}^{n-d}, M\left(x_{0}^{\prime \prime}\right) \equiv$ $\left\{x^{\prime}:\left(x^{\prime}, x_{0}^{\prime \prime}\right) \in M\right\}$ is a Lipschitz domain in $\mathbf{R}^{d}$. Moreover there is a rotation of the $x^{\prime}$ coordinates to $y^{\prime}=\left(\hat{y}, y_{d}\right) \in \mathbf{R}^{d-1} \times \mathbf{R}^{1}$ and a function $g\left(\hat{y}, x^{\prime \prime}\right)$ defined for almost every $x^{\prime \prime}$, Lipschitz continuous in $\hat{y}$ (with Lipschitz constant independent of $\left.x^{\prime \prime}\right)$, so that

$$
M\left(x^{\prime \prime}\right)=\left\{y^{\prime} \in \mathbf{R}^{d}: y_{d}<g\left(\hat{y}, x^{\prime \prime}\right)\right\} \text {. }
$$

LEMMA 4.5. Suppose $u_{0}(x)$ is admissible and piecewise continuous. Let $\tilde{u}$ be as in Lemma 4.1 but with $x^{\prime}$ replaced by the rotated coordinates, $y^{\prime}=\left(\hat{y}, y_{d}\right)$. Then for almost every $x^{\prime \prime} \in \mathbf{R}^{n-d}$ and any $\delta>0$ there is a constant $T\left(\delta, x^{\prime \prime}\right)<\infty$ so that

$$
\begin{array}{lll}
\tilde{u}\left(t, y^{\prime} ; x^{\prime \prime}\right)=a & \text { for a.e. } y \text { such that } \quad y_{n} \geq g\left(\hat{y}, x^{\prime \prime}\right)+\delta, t \geq T, \\
\tilde{u}\left(t, y^{\prime} ; x^{\prime \prime}\right)=b & \text { for a.e. } y \text { such that } \quad y_{n} \leq g\left(\hat{y}, x^{\prime \prime}\right)-\delta, t \geq T .
\end{array}
$$

ProOF. Assume that $f(a)=f(b)=0$, so that $k=0$ and $u=\tilde{u}$. Consider for each $j=1,2, \ldots, q_{1 / j}$ and $p_{1 / j}$ as defined in Lemma 3.7. For $t \geq t^{*}$, usirg Theorem 2.3,

$$
a \leq q_{1 / j}(t, x) \leq p_{1 / j}(t, x) \leq b .
$$

Now there exists a set $Z$ of full $\mathcal{L}^{n-d}$ measure so that for all $j$,

$$
q_{1 / j}\left(t^{*}, x^{\prime}, x^{\prime \prime}\right) \leq u\left(t^{*}, x^{\prime}+h, x^{\prime \prime}\right) \leq p_{1 / j}\left(t^{*}, x^{\prime}, x^{\prime \prime}\right)
$$

for $\mathcal{L}^{d}$ a.e. $x^{\prime}$, for each $|h|<\tau(1 / j)$ and $x^{\prime \prime} \in Z$. By construction $p_{1 / j}\left(t^{*}, x\right)-$ $q_{1 / j}\left(t^{*}, x\right) \rightarrow 0$ as $j \rightarrow \infty$ in $L_{\text {loc }}^{1}\left(\mathbf{R}^{n}\right)$. Hence for almost every $x^{\prime \prime} \in Z$,

$$
\int_{\left|x^{\prime}\right| \leq R}\left[p_{1 / j}\left(t^{*}, x^{\prime}, x^{\prime \prime}\right)-q_{1 / j}\left(t^{*}, x^{\prime}, x^{\prime \prime}\right)\right] d x^{\prime} \rightarrow 0
$$

as $j \rightarrow \infty$ for any $R<\infty$. Using Lemma 4.1 we can argue as in Lemma 3.7 and Theorem 3.11 for each such $x^{\prime \prime}$ to obtain the conclusion of the lemma.

LEMMA 4.6. Let $u_{0}(x)$ be admissible and piecewise continuous. Then for all $\varepsilon>0$ and almost every $z^{\prime \prime} \in \mathbf{R}^{n-d}$ there is a constant, $T\left(\varepsilon, z^{\prime \prime}\right)$, such that if 


$$
\begin{aligned}
& x^{\prime \prime}-k^{\prime \prime} t=z^{\prime \prime}, \\
& \begin{aligned}
u\left(t, x^{\prime}, x^{\prime \prime}\right) & =\tilde{u}\left(t, x^{\prime}-k^{\prime} t ; x^{\prime \prime}-k^{\prime \prime} t\right) \\
& =b \cdot \chi_{M\left(x^{\prime \prime}-k^{\prime \prime} t\right)}\left(x^{\prime}-k^{\prime} t\right)+a \cdot \chi_{R^{d}-M\left(x^{\prime \prime}-k^{\prime \prime} t\right)}\left(x^{\prime}-k^{\prime} t\right)
\end{aligned}
\end{aligned}
$$

for $t \geq T$ and almost every $x^{\prime}$ such that $\operatorname{dist}\left(x^{\prime}-k^{\prime} t, \partial M\left(x^{\prime \prime}-k^{\prime \prime} t\right)\right)>\varepsilon$.

\section{REFERENCES}

1. E. Conway, The formation and decay of shocks for a conservation law in general dimensions, Arch. Rational Mech. Anal. 64 (1977), 47-57.

2. C. M. Dafermos, Characteristics in hyperbolic conservation laws. A study of the structure and the asymptotic behavior of solutions, Research Notes in Mathematics, Nonlinear Analysis and Mechanics: Heriot-Watt Symposium, Vol. 4, (R. J. Knops, Ed.), Pitman, 1979.

3. E. Giusti, Minimal surfaces and functions of bounded variation, Notes on Pure Mathematics, Australian National University, Canberra, 1977.

4. A. M. Ilin and O. A. Oleinik, Behavior of the solutions of the Cauchy problem for certain quasilinear equations for unbounded increase of time, Dokl. Akad. Nauk SSSR 120 (1958), 25-28; English transl., Amer. Math. Soc. Transl. (2) 42 (1964), 19-23.

5. S. N. Kruzkov, First order quasilinear equations with several independent variables, Math USSR-Sb. 10 (1970), 217-273.

6. W. B. Lindquist, The scalar Riemann problem in two spatial dimensions: piecewise smoothness of solutions and its breakdown, preprint (1984).

7. T. P. Liu, Invariants and asymptotic behavior of solutions of a conservation law, Proc. Amer. Math. Soc. 71 (1978), 227-231.

8. A. I. Vol'pert, The space BV and quasilinear equations, Math. USSR-Sb. 2 (1967), 225267.

9. D. H. Wagner, The Riemann problem in two space dimensions for a single conservation law, SIAM J. Math. Anal. 14 (1983), 534-559.

Department of Mathematics, Purdue University, West lafayette, Indiana 47907 